hep-th/0010144

SLAC-PUB-8671

\title{
Gauge Fields, Scalars, Warped Geometry, and Strings
}

\author{
Eva Silverstein \\ Department of Physics and SLAC \\ Stanford University \\ Stanford, CA 94305/94309
}

\begin{abstract}
We review results on several interesting phenomena in warped compactifications of M theory, as presented at Strings 2000. The behavior of gauge fields in dimensional reduction from $d+1$ to $d$ dimensions in various backgrounds is explained from the point of view of the holographic duals (and a point raised in the question session at the conference is addressed). We summarize the role of additional fields (in particular scalar fields) in $5 \mathrm{~d}$ warped geometries in making it possible for Poincare-invariant domain wall solutions to exist to a nontrivial order in a controlled approximation scheme without fine-tuning of parameters in the $5 d$ action (and comment on the status of the singularities arising in the general relativistic description of these solutions). Finally, we discuss briefly the emergence of excitations of wrapped branes in warped geometries whose effective thickness, as measured along the Poincare slices in the geometry, grows as the energy increases.
\end{abstract}

October 2000 


\section{Introduction}

Generic general-relativistic spacetime backgrounds with $d$-dimensional Poincare invariance have a metric of the form

$$
e^{2 A(y)} \eta_{\mu \nu} d x^{\mu} d x^{\nu}+H_{I J}(y) d y^{I} d y^{J}
$$

where $\mu, \nu=0, \ldots, d-1$. Canonical examples in M theory with a nontrivial warp factor $e^{2 A(y)}$ include heterotic compactifications with $(0,2)$ worldsheet supersymmetry [1], compactified Horava-Witten theory [2], $A d S_{d+1}$ and its relevant deformations, linear dilaton theories such as for example the NS5-brane solution [3] and the conifold singularity [4] [河, and no doubt many more solutions yet to be discovered with less supersymmetry.

Many warped backgrounds have non-gravitational holographic duals [6], but most have no known equivalent "boundary theory". Almost all of these backgrounds have curvature singularities and/or strong coupling at some finite proper distance from a generic points on the component of the geometry parameterized by $y^{I}$, so that general relativity breaks down in this region of the background.

It is important and interesting to understand as precisely as possible the physics of this type of background, in particular to see if any new phenomena emerge from the warped shape of the spacetime. In this talk I will review results on three aspects of this physics:

(1) The behavior of gauge fields

(2) The role of for example scalar fields in making possible, to the leading order in a controlled approximation scheme, solutions with Poincare invariance even after some nontrivial quantum corrections to the vacuum energy have been included, and

(3) The behavior of massive states coming from wrapped branes in this sort of geometry: in particular one finds a new corner of the theory where excitations can be seen to grow in size as they grow in energy as a consequence of the warping in the metric (1.1).

\section{Gauge Fields}

If we focus on cases where the warping occurs along a single direction $y$, the low energy effective action (to the extent that it is reliable) takes the form

$$
S=\int d^{d} x d y \sqrt{g}\left(a(\phi) R+b(\phi)(\nabla \phi)^{2}+c(\phi) F^{2}-\Lambda(\phi)\right) .
$$


If the integral over $y$ of the Einstein term is finite one obtains a finite $d$-dimensional Planck scale and "trapped gravity" [7]. This holds also for a $d$-dimensional graviphoton that arises from a $d+1$-dimensional two-form potential, as demonstrated recently in [8]. On the other hand if there is a $d+1$-dimensional vector potential, the dimensional reduction of its kinetic term might give a divergence independent of what is going on with the graviton kinetic term. In particular in the $\int d^{d} x d y F^{2}$ term there are two powers of the inverse $d+1$ dimensional metric as opposed to the single power of $g^{\mu \nu}$ in the dimensional reduction of the Einstein term.

The coefficient of the $d$-dimensional gauge kinetic term $\frac{1}{e_{d}^{2}} \int d^{d} x t r F^{2}$ is the inverse effective gauge coupling (charge) squared of the $d$-dimensional gauge theory. If the effective gauge coupling in $d$-dimensions is zero, then one might naively infer that this symmetry behaves like a global symmetry rather than a gauge symmetry after dimensional reduction. This would be very surprising since the black hole no-hair theorems, at least in contexts where they have been studied, indicate that information about global charges is lost in processes in which the black hole absorbs particles which carried this charge. On the other hand in this context, where the symmetry is a bona fide gauge symmetry in $d+1$ dimensions, the charges must be conserved.

In [9] we found that in several known examples of warped geometries in which $\frac{1}{e_{d}^{2}}$ diverges, this divergence is either indicative of a conventional screening effect or the effective theory (2.1) breaks down at some $y<\infty$ where new behavior takes over that also has a conventional behavior in the IR of the $d$-dimensional description of the physics.

The simplest example is the cutoff $A d S_{5}$ background studied by Randall and Sundrum [7]. In this background, $A(y)=-|y| / L$ where $L$ is the curvature radius of AdS. The calculation giving the effective charge in $4 d$ is

$$
\frac{1}{e_{4}^{2}} \sim \frac{1}{e_{5}^{2}} \int_{0}^{y_{0}} d y e^{-4 y / L}\left(e^{2 y / L}\right)^{2} \sim y_{0}
$$

where we have introduced an IR momentum cutoff $p_{0}$ through the relation $y_{0} \sim \operatorname{Llog}\left(p_{0} L\right)$ following from the metric. The first factor in the integrand comes from $\sqrt{g}$, and the last from the two powers of the inverse metric involved in forming $\operatorname{tr} F^{2}$.

So we are finding

$$
\frac{1}{e_{4}^{2}} \sim \frac{1}{e_{5}^{2}} \operatorname{Llog}\left(p_{0} L\right)
$$

This logarithmic IR divergence is the result one would expect from screening of the charge from the $4 d$ description (as first pointed out to us by E. Witten, and as first noted in 
papers of Pomaral [10]). This interpretation is confirmed by an explicit calculation of the electrostatic potential arising from a point source of charge at $y=0$ :

$$
A_{0}(p, y=0)=-\frac{Q K_{1}(p L)}{2 K_{0}(p L)} \rightarrow_{p \rightarrow 0} \frac{Q}{p^{2} \log p}
$$

Similarly one finds a generalization of the screening effect to higher dimensions and higher-form fields. In dimension $d$, for a $q$-form field strength, as a function of IR momentum cutoff $p_{0}$, we find

$$
\begin{gathered}
\frac{1}{e_{q}^{2}} \propto \quad e^{\frac{R}{L}(2 q-d)} \sim \frac{1}{p_{0}^{2 q-d}} \quad 2 q \neq d \\
R \sim \log \left(p_{0}\right) \quad 2 q=d
\end{gathered}
$$

At the conference, M. Duff asked about the consistency of this result with the possibility of dualizing $q$-form field strengths to $5-q$-form field strengths. For example a scalar field $\eta$ with a 1 -form field strength would be dual to a 3 -form potential field $C$ with a 4-form field strength. The latter, from (2.5), gives a mode with zero charge upon dimensional reduction; whereas a scalar field, like gravity, is left with nontrivial interactions after dimensional reduction.

I believe the answer to this is as follows (this result was obtained in collaboration with M. Schulz). The equation for dualizing a form, for example $d \eta=* d C$, is a linear differential equation which locally has a solution. There is no guarantee, however, that this solution is nonsingular everywhere.

Consider the equation for a scalar field in the background (1.1). A massless mode in $4 d$ satisfies the equation

$$
\eta^{\prime \prime}+4 \eta^{\prime} A^{\prime}=0
$$

where primes denote differentiation with respect to $y$. One obvious solution is the zero mode, $\eta=\eta(x)$ independent of $y$. This is the mode which gets "trapped" upon dimensional reduction, with a finite kinetic term. As noted by Duff in his question, this cannot be the solution dual to the three-form potential.

There is another solution to (2.6), which becomes singular at the AdS horizon in the RS geometry. Integrating (2.6), this solution satisfies

$$
\eta^{\prime}=\hat{\eta}(x) e^{-4 A(y)}
$$


For the RS geometry, this yields

$$
\eta \sim \hat{\eta}(x) e^{+4 y / L}
$$

The dimensional reduction of the kinetic term for this scalar goes like

$$
\int_{0}^{y_{0}} d y e^{-4 y / L}\left(e^{4 y / L}\right)^{2} \sim e^{4 y_{0} / L}
$$

from one power of $\sqrt{g}$ and two powers of $e^{+4 y / L}$ from the solution (2.8) appearing in the quadratic action for $\eta$.

This is the same divergence which arises for the dimensional reduction of the standard zero-mode solution for the three-form potential $C$, which goes like

$$
\int_{0}^{y_{0}} d y e^{-4 y / L}\left(e^{2 y / L}\right)^{4}
$$

from one power of $\sqrt{g}$ and four powers of the inverse metric required to form the square of the four-form field strength. So the second solution (2.8) for the scalar is evidently the one dual to the zero-mode of the three-form potential.

Consider the $\mathrm{N}$-NS 5-brane solution of type II string theory [3]. It has a string-frame metric and dilaton

$$
\begin{aligned}
& d s^{2}=d x_{6}^{2}+d r^{2}+l_{s}^{2} N d \Omega_{3}^{2} \\
& \phi=\alpha r
\end{aligned}
$$

with $\alpha=1 / l_{s} \sqrt{N}$.

The string-frame ten-dimensional action is

$$
\int d^{6} x d r d \Omega_{3}\left[e^{-2 \phi}\left(R+(\partial \phi)^{2}\right)+K_{R R}^{2}\right]
$$

where $K$ is the field strength for the RR U(1) gauge field of type IIA string theory, or the field strength for the 2-form RR gauge potential of type IIB string theory.

In the dimensional reduction, $6 d$ gravity survives (the $6 d$ Planck scale ending up finite because of the coupling of the Einstein term to $e^{-2 \phi}$ in string frame). On the other hand the RR gauge field effectively propagates in seven flat dimensions according to the metric (2.11) since its kinetic term is independent of $\phi$. This is not a screening phenomenon in $6 d$.

The resolution is that the breakdown of the solution (2.11) down the throat due to strong coupling effects is important. The IIA NS5-brane is fundamentally an M5-brane 
at a point on the eleventh circle of $M$ theory [11][12]. This means that deep in the IR region of the solution the $\mathrm{RR}$ gauge symmetry is spontaneously broken, and this Higgs mechanism wards off the more exotic possibility of a conserved global symmetry persisting in $6 d$.

These examples provide further evidence for the robustness of the arguments against global symmetries in quantum gravity. However, it is interesting to keep one's eyes out for more exotic examples that might arise in which a naive divergence in the dimensionally reduced gauge kinetic term persists into the infrared in a way that cannot be understood from screening or the Higgs mechanism. In such a background, the black-hole no-hair theorems would need to be analyzed carefully.

\section{Scalars}

Let us focus now on the physics of gravity plus scalars, with action in a $5 d$ bulk and on a $4 d \delta$-function localized brane given by

$$
\begin{aligned}
S= & \int d^{5} x \sqrt{-G}\left[R-\frac{4}{3}(\nabla \phi)^{2}-\Lambda e^{a \phi}\right] \\
& +\int d^{4} x \sqrt{-g}(-f(\phi))
\end{aligned}
$$

We have taken the bulk $\Lambda$ to be zero to a leading approximation, having in mind bulk supersymmetry which is only broken at the level of interactions with the brane.

In contrast to the Randall-Sundrum system, this one has $4 d$ Poincare-invariant solutions for generic brane tension $f(\phi)$ [13]. The solutions in the bulk are:

$$
\begin{gathered}
\phi(y)= \pm \frac{3}{4} \log \left|\frac{4}{3} y+c\right|+d \\
A(y)=\frac{1}{4} \log \left|\frac{4}{3} y+c\right|+\tilde{d}
\end{gathered}
$$

At $y=-\frac{3}{4} c$, this configuration has curvature singularities: general relativity breaks down near these points.

Einstein's equations at the wall at $y=0$ (which boil down to Israel matching conditions there) are solved by adjusting the values of integration constants $c, d$, not by tuning parameters like $f(\phi)$ in the Lagrangian. So quantum corrections to $f(\phi)$ will not ruin the fact that there is a flat solution. This is perhaps encouraging, since in $\mathrm{M}$ theory all indications are that one does not have the freedom to tune parameters in the theory (there 
being no arbitrary dimensionless couplings put in); we at most have the freedom to choose among different solutions of the basic equations (still to be determined!) of the theory.

It is therefore an interesting goal to exhibit as a first step a background of $M$ theory with near-vanishing cosmological term after nontrivial quantum corrections are included, as also noted recently in [14]. The next (and presumably most difficult) step is to understand why we live in such a background as opposed to one of the millions of others with large spacetime curvatures or otherwise unrealistic low-energy physics.

In our examples (3.1) (3.2) (3.3), we must first understand the physics of the singularities that appear in the general-relativistic description of the system. Progress was made on this front in two directions. Firstly, Horowitz, Low, and Zee found stringy cosmological solutions with again effectively zero cosmological term independent of the parameters in the Lagrangian [15]. In these solutions, there are again curvature singularities but they are null, so that there is no issue of additional boundary conditions needed for modes emanating outward from the brane.

Secondly, the static solutions above are in some ways analogous to the types of backgrounds that have been intensely studied recently as deformations of the AdS/CFT correspondence to confining theories (see for example [16]). In those backgrounds, a singularity in the general relativistic approximation corresponds to the presence of a mass gap beyond which there are no excitations of the field theory. Just as the presence of a mass gap is generic to asymptotically free quantum field theory, the presence of a singularity in the $5 d$ gravity dual in the (bad) general relativistic approximation is generic. That is not to say that all singular backgrounds of this form are resolved by quantum gravity effects. Indeed, in this AdS/CFT context the field theory side can only vouch for a discrete subset of the continuum of apparent singular solutions in the GR description, since at finite $N$ the field theory has a finite number of vacua. Luckily there are of order $e^{\sqrt{N}}$ such vacua.

So the upshot, in this context, is this. Couple a large-N gauge theory to the standard model in the way that is determined by the addition of a thin domain wall to cut off the UV end of the gravity dual to the gauge theory. Generically, one can choose a vacuum of the large- $\mathrm{N}$ theory to zero the $4 d$ cosmological term in the full system, up to corrections that are parametrically suppressed relative to the $\mathrm{TeV}^{4}$ contribution expected from standard model loops. These corrections could also introduce instabilities in the system, which is a problem endemic to these models as well as those of [7](not to mention any string compactification with approximate moduli). 
Is there any reason this vacuum is preferred? Aside from the partially anthropic arguments presented in [17] 18] (where another interesting procedure for finding solutions of M theory with near-vanishing cosmological term was obtained) I know of no argument for this at present. One advantage of our setup in the context of the Brown-Teitelboim style analysis of [17] is that the "discretuum" of different vacua in our case all have manifestly similar "standard model" physics since this resides on the brane and the bulk is where the cancellation mechanism arises. As emphasized in [19], this is an important consideration in entertaining this sort of anthropic explanation for the history.

However, it is worth emphasizing that the actual solutions (3.2) (3.3) considered in [13] do not asymptote to AdS space in bulk, but instead tend toward flat space far from the singularity. Therefore they are not dual to a quantum field theory in toto. It is possible that the regime near the singularity does have an effective quantum field theory dual, since the warp factor decreases there [20]. In any case it is very interesting to try to understand the physics of warped geometries which do not have a quantum field theory dual. Related to this is the question of whether any backgrounds of this type can have sensible physics in which effective quantum field theory breaks down so that long-distance quantities like the cosmological constant might be affected naturally by high-energy excitations in the theory.

I cannot resist adding here more general comments on naked singularities in string theory. At such singularities, general relativity breaks down. To me this is one of the most interesting features in a spacetime, since it is an opportunity to learn about physics of $\mathrm{M}$ theory that goes beyond the long-wavelength general relativistic approximation. The resolution of singularities on the Coulomb branch of gauge theories in Seiberg-Witten theory [21], in ADE limits of compactifications of type II string theory on K3 [22], at type II conifold points [23] and their heterotic cousins 24] 25] 26] (just to name a few examples), involved in a detailed way the physics of non-perturbative excitations of the theory and nontrivial information about its strong-coupling behavior. Without these many resolutions of general-relativistic naked singularities, the web of dualities relating different limits of M theory would not exist. One of the immediate applications of such resolutions has also been the analysis of controlled topology-changing transitions [5] [27] 26] in M theory.

For the most general solutions described in this section, it remains to be seen whether or not the singularities have a (perhaps discretized) resolution, and if so whether a standard $4 d$ effective field theory arises at long distances. (In the above examples, on the order of a decade passed between the original nakedly singular solutions being written down (for 
the conifold for example in [4] ) and the eventual quantum resolution; it is not clear in our case when all the necessary ingredients will be available to answer this question.) This is an interesting open question.

\section{Wrapped Branes}

One of the features of quantum gravity emphasized recently for example in [28] 29] is the fact that at the highest energies, excitations (namely black holes) grow in size as they grow in energy (mass). This is in stark contrast to the size $=1 /$ momentum uncertainty relation of elementary excitations in quantum field theory. This makes it conceivable that such high-energy excitations could naturally affect long-distance parameters.

It turns out that warped compactifications constitute another context in which some excitations grow in size as they grow in energy. In general given a metric (1.1), there will be wrapped branes on the compact component of the geometry whose mass $m_{0}(y)$ will depend on $y$ since the volume of the cycle on which the brane is wrapped varies as a function of $y$. As measured along the Poincare slices, the energy will be (taking into account the warp factor as well as the variation of the cycle volume)

$$
E(y) \sim \sqrt{g_{00}(y)} m_{0}(y)
$$

On the other hand, because of the warp factor, the thickness of the object is rescaled from its proper thickness $r_{0}$ because of the warp factor:

$$
R \equiv \delta x_{\|} \sim \frac{1}{\sqrt{g_{i i}}} r_{0}
$$

In 30] concrete examples were exhibited in which both $R$ and $E$ grow in the same direction, so that

$$
E=T R^{q}
$$

for some power $q>0$. The spectrum of excitations of these wrapped branes was worked out in a limit where a Kaluza-Klein analysis was valid.

Any situation in which there is this sort of growth of size with energy suggests that interesting nonlocal effects might emerge from the dynamics of these excitations. This is under investigation 31.

\section{Acknowledgements}


The work in $\S 2$ was done in collaboration with N. Kaloper and L. Susskind. The work reviewed in $\S 3$ was done in collaboration with S. Kachru and M. Schulz, and concurrently by N. Arkani-Hamed, S. Dimopoulos, N. Kaloper, and R. Sundrum. M. Duff asked an interesting question about the material in $\S 2$. I thank all of the above, as well as those mentioned in the corresponding papers, for many interesting discussions on these and related topics. Finally I would like to thank the organizers of Strings 2000 for a stimulating conference. This work was supported by a DOE OJI grant, by the A.P. Sloan Foundation, and by the DOE under contract DE-AC03-76SF00515. 


\section{References}

[1] A. Strominger, "Superstrings With Torsion," Nucl. Phys. B274, 253 (1986).

[2] E. Witten, "Strong Coupling Expansion Of Calabi-Yau Compactification," Nucl. Phys. B471, 135 (1996) hep-th/9602070.

[3] A. Strominger, "Heterotic Solitons," Nucl. Phys. B343, 167 (1990); C. G. Callan, J. A. Harvey and A. Strominger, "Supersymmetric string solitons," hep-th/9112030.

[4] P. Candelas and X. C. de la Ossa, "Comments On Conifolds," Nucl. Phys. B342, 246 (1990).

[5] E. Witten, "Phases of $\mathrm{N}=2$ theories in two dimensions," Nucl. Phys. B403, 159 (1993) hep-th/9301042.

[6] J. Maldacena, "The large $\mathrm{N}$ limit of superconformal field theories and supergravity," Adv. Theor. Math. Phys. 2, 231 (1998), hep-th/9711200; E. Witten, "Anti-de Sitter space and holography," Adv. Theor. Math. Phys. 2, 253 (1998) hep-th/9802150; S. S. Gubser, I. R. Klebanov and A. M. Polyakov, "Gauge theory correlators from non-critical string theory," Phys. Lett. B428, 105 (1998), hep-th/9802109.

[7] L. Randall and R. Sundrum, "An Alternative to Compactification" Phys. Rev. Lett. 83, 4690 (1999), hep-th/9906064

[8] H. Lu and C. N. Pope, "Branes on the brane," hep-th/0008050. M. Cvetic, H. Lu and C. N. Pope, "Brane-world Kaluza-Klein reductions and branes on the brane," hep-th/0009183. M. J. Duff, J. T. Liu and W. A. Sabra, "Localization of supergravity on the brane," hep-th/0009212;

[9] N. Kaloper, E. Silverstein, and L. Susskind, "Gauge symmetry and localized gravity in M theory," hep-th/0006192.

[10] A. Pomarol, "Grand unified theories without the desert," hep-ph/0005293.

[11] N. Itzhaki, J. Maldacena, J. Sonnenschein, and Shimon Yankielowicz, "Supergravity and the Large N Limit of Theories with Sixteen Supercharges", hep-th/9802042.

[12] S. Minwalla and N. Seiberg, "Comments on the IIA NS5-brane," JHEP 9906, 007 (1999), hep-th/9904142.

[13] S. Kachru, M. Schulz, E. Silverstein Phys. Rev. D62, 045021 (2000) hep-th/0001206; N. Arkani-Hamed, S. Dimopoulos, N. Kaloper and R. Sundrum, "A small cosmological constant from a large extra dimension," Phys. Lett. B480, 193 (2000) hep-th/0001197.

[14] S. P. de Alwis, "Brane world scenarios and the cosmological constant," hepth/0002174.

[15] G. Horowitz, I. Low, and A. Zee, "Self-tuning in an outgoing brane wave model," Phys. Rev. D62, 086005 (2000) hep-th/0004206.

[16] L. Girardello, M. Petrini, M. Porrati and A. Zaffaroni, "Novel local CFT and exact results on perturbations of $\mathrm{N}=4$ super Yang-Mills from AdS dynamics," JHEP 9812, 022 (1998) hep-th/9810126; D. Z. Freedman, S. S. Gubser, K. Pilch and N. P. Warner, 
"Renormalization group flows from holography supersymmetry and a c-theorem," hepth/9904017; J. Polchinski and M. J. Strassler, "The string dual of a confining fourdimensional gauge theory," hep-th/0003136.

[17] R. Bousso and J. Polchinski, "Quantization of four-form fluxes and dynamical neutralization of the cosmological constant," JHEP 0006, 006 (2000) hep-th/0004134.

[18] J. L. Feng, J. March-Russell, S. Sethi and F. Wilczek, "Saltatory relaxation of the cosmological constant," hep-th/0005276.

[19] T. Banks, M. Dine and L. Motl, "On anthropic solutions of the cosmological constant problem," hep-th/0007206.

[20] J. Maldacena and C. Nunez, "Supergravity description of field theories on curved manifolds and a no go theorem," hep-th/0007018.

[21] N. Seiberg and E. Witten, "Electric - magnetic duality, monopole condensation, and confinement in N=2 supersymmetric Yang-Mills theory," Nucl. Phys. B426, 19 (1994) hep-th/9407087.

[22] E. Witten, "String theory dynamics in various dimensions," Nucl. Phys. B443, 85 (1995) hep-th/9503124.

[23] A. Strominger, "Massless black holes and conifolds in string theory," Nucl. Phys. B451, 96 (1995) hep-th/9504090.

[24] E. Witten, "Small Instantons in String Theory," Nucl. Phys. B460, 541 (1996) hepth/9511030.

[25] S. Kachru, N. Seiberg and E. Silverstein, "SUSY Gauge Dynamics and Singularities of 4d N=1 String Vacua," Nucl. Phys. B480, 170 (1996) hep-th/9605036.

[26] S. Kachru and E. Silverstein, "Chirality-changing phase transitions in 4d string vacua," Nucl. Phys. B504, 272 (1997) hep-th/9704185.

[27] P. S. Aspinwall, B. R. Greene and D. R. Morrison, "Calabi-Yau moduli space, mirror manifolds and spacetime topology change in string theory," Nucl. Phys. B416, 414 (1994) hep-th/9309097; J. Distler and S. Kachru, "(0,2) Landau-Ginzburg theory," Nucl. Phys. B413, 213 (1994) hep-th/9309110; B. R. Greene, D. R. Morrison and A. Strominger, "Black hole condensation and the unification of string vacua," Nucl. Phys. B451, 109 (1995) hep-th/9504145.

[28] J. McGreevy, L. Susskind, N. Toumbas, "Invasion of the giant gravitons from anti-de Sitter space," JHEP 0006, 008 (2000) hep-th/0003075.

[29] T. Banks, hep-th/0007146.

[30] E. Silverstein, "Extended States from Warped Compactifications of M Theory", hepth/0009057.

[31] A. Adams and E. Silverstein, work in progress. 